Review

\title{
DNA vaccines and recombinant allergens with reduced allergenic activity treat allergies
}

\author{
Stanislava Yu. Petrova ${ }^{1}$, Svetlana V. Khlgatian ${ }^{1,2}$, Elena V. Svirshchevskaya ${ }^{3}$, Anna V. Vasilyeva ${ }^{1}$, \\ Valentina M. Berzhets ${ }^{1}$ \\ ${ }^{1}$ I.I. Mechnikov Research Institute for Vaccines and Sera, Moscow, Russia \\ ${ }^{2}$ Research Center of Neurology, Moscow, Russia \\ ${ }^{3}$ M.M. Shemyakin - Yu.A. Ovchinnikov Institute of Bioorganic Chemistry, Russian Academy of Sciences, Moscow, Russia
}

Received 10 February 2020, Revised 30 April 2020, Accepted 3 August 2020

(C) 2020, Petrova S.Yu., Khlgatian S.V., Svirshchevskaya E.V., Vasilyeva A.V., Berzhets V.M.

(C) 2020, Russian Open Medical Journal

\begin{abstract}
This review is intended to familiarize readers with major novel directions of developing allergy vaccines, their structure, as well as the mechanisms of forming a new immunological response in the course of the treating immunoglobulin $\mathrm{E}$ (IgE)-mediated allergic diseases. Currently, science offers a huge variety of new experimental forms of recombinant allergens with reduced allergenic activity and increased immunogenicity, or vice-versa, immune tolerance. Often, the mechanisms of their effect on the immune system are not fully understood. Scientific publications, including reviews covering this topic, allowed us identifying top priority areas in the development of allergy vaccines: recombinant hypoallergenic allergen derivatives, $T$ cell epitope-based allergy vaccines, and B cell epitope-based allergy vaccines. In addition, the review discusses use of deoxyribonucleic acid (DNA) vaccines. Immunotherapy with DNA vaccines is the newest and least studied method of treating allergic diseases.
\end{abstract}

Keywords: recombinant hypoallergenic allergen derivatives, T cell epitope-based allergy vaccines, B cell epitope-based allergy vaccines, encapsulated allergens, DNA vaccines.

Cite as Petrova S.Yu., Khlgatian S.V., Svirshchevskaya E.V., Vasilyeva A.V., Berzhets V.M. DNA vaccines and recombinant allergens with reduced allergenic activity treat allergies. Russian Open Medical Journal 2021; 10: e0102.

Correspondence to Stanislava Yu. Petrova. Address: 5a, Maly Kazennyi pereulok, Moscow 105064, Russia. Phone: +7 9164633297. E-mail: laball@yandex.ru.

\section{Introduction}

Immunoglobulin E (IgE)-mediated reactions underlie the pathogenesis of many allergic diseases caused by genetic disorders and/or an influence of the damaging effect of environmental factors on immune system functioning. Pathological process development leads to functional and/or quantitative changes in the ratios of interconnected cell populations in adaptive link of the immunity. A major immunological shift is traced in the imbalance of regulatory impacts among T-helper 1 (Th1), T-helper 2(Th2) and Treg cells, with Th2 prevailing. Immunopathological shifts result in changes in the ratios of cytokines IL4/IFN- $\gamma$ (Interleukin-4 /interferon-gamma) and IL4/IL10 (Interleukin-4 / Interleukin-10) with a shift towards IL4. Ultimately, these disorders lead to a switch in the synthesis of antibodies to IgE in B cells, and the launch of further immunological, pathochemical and pathophysiological stages of allergic processes, resulting in damage to body tissues [1, 2].

Allergen-specific immunotherapy (ASIT) is the only example of antiallergic treatment affecting pathogenetic links of the IgEdependent allergic process. After the completion of the treatment course, ASIT, has a long-term prophylactic effect, possibly caused by a change in the nature of the body immune response to the allergen $[1,3,4]$. The mechanisms of ASIT action include very early effects of desensitization, inhibition of migration and suppression of the mediator release from eosinophils, basophils and mast cells $[6,7]$, as well as the mechanisms of modulation of T-cell and B-cell responses and associated antibody isotypes $[3,5]$.

The widespread introduction of recombinant technologies and the production of allergen proteins opens up new opportunities for ASIT [6, 7]. At present, using recombinant deoxyribonucleic acid (DNA) technology, it is possible to obtain safe vaccines against allergies. Such approaches allow overcoming the problems of specificity and high allergenic activity associated with the use of natural allergens [8-10].

The reviews by domestic and foreign authors consider a wide array of approaches to development of new generation allergy vaccines and immunization schemes based on them. In particular, R. Valenta, M. Akdis, K. Marth and other researchers provide information about structural features and possible mechanisms of forming new immunological responses in the course of treating IgE-mediated allergic diseases with contemporary allergy vaccines [4, 9-14]. Numerous recently published studies devoted to the success in the development and use of allergy vaccines dictate the need for systematization, generalization and analysis of substantial available information flow.

The goal of this review is a comprehensive analysis of accumulated knowledge concerning major directions of developing novel vaccines against allergy. In addition, the review 
discusses DNA vaccines used for immunotherapy, which is the newest and least studied method of treating allergic diseases.

\section{B- and T-cell protein epitopes}

In order to navigate the huge variety of new experimental forms of allergens and understand the mechanism of their effect on immune system, numerous studies have been carried out to investigate the structure of protein molecules of allergens, primarily in order to determine the localization of B-cell epitopes in them and decipher their conformational organization. It is known that B-cell antigenic determinants are able to bind to antibodies, including IgE. B-cell epitopes are usually located on the outer surface of the allergen [15], and they can be classified into two groups: linear and conformational. Linear epitopes are formed by linear stretches of amino acid sequences of proteins. Conformational epitopes are formed by sections of amino acid sequences, located far from each other and forming a tertiary structure by folding a protein molecule. Despite the fact that nearly $90 \%$ of the epitopes are conformational, some of them contain one or several linear regions. Linear epitopes of B cells are composed of peptides that are easily synthesized in the laboratory. Therefore, despite the fact that they are in the minority, much attention is paid to identification of linear B-cell epitopes [16-18]. Allergens have at least two IgE antibody-binding epitopes that can "stitch" two membrane-bound IgEs [4]. Mature $B$ cells can also directly contact antigen B-cell epitopes via specific immunoglobulins (B-cell receptors, or BCRs) located on their surface. BCRs enable them to specifically recognize, bind, process and present foreign allergen molecules to the Th2 cell, along with the major histocompatibility complex (MHC) of class II. Th activate $B$ cells for the synthesis of antibodies [16-19].

Alternatively, T cells have TCR receptors on their surface that, in conjunction with $\mathrm{MHC}$, are capable of recognizing foreign antigens presented on the surface of various antigen-presenting cells (APCs). The T-cell epitope of an allergen is a linear peptide fragment that binds to the $\mathrm{MHC}$ and is subsequently recognized by the $\mathrm{T}$ lymphocyte. Prediction of T-cell epitopes is aimed at identifying the shortest peptides in the antigen able to stimulate $T$ cells $[18,20]$.

Currently, several promising trends in the development of vaccines against allergy can be identified: hypoallergenic derivatives of recombinant allergens, allergy vaccines based on the $\mathrm{T}$-cell epitope, and allergy vaccines based on the B-cell epitope.

Trend 1: Development of recombinant hypoallergenic allergen derivatives

To obtain hypoallergenic derivatives of recombinant allergens, various procedures can be used: the protein can be fragmented, and the amino acid sequences in it can be interchanged; mutations, aimed at certain structures of the protein, can be carried out; hybrid proteins can be created from the fragments of different proteins; change the spatial orientation of the protein molecule can be changed; finally, fragments of the allergen can be reassembled in the form of a mosaic [9-11, 13, 21]. When vaccinated with recombinant allergens of this type, the binding of the antigen to the reagins decreases, but the formation of IgG to remaining unchanged peptides on the protein surface is preserved. This is due to the fact that the body has a wide variety of B-cell receptors, enabling immune system to normally recognize all naturally occurring pathogens. In the course of immunization, the specificity and affinity of the antibodies produced by the body increase. As a result, during the immune response, blocking IgGs are obtained, which prevent the binding of the wild-type allergen to the IgE of the patients with allergies [15]. The latter fact leads to the absence or reduction of IgE-mediated reactions of the immediate type throughout immunotherapy. Despite the fact that hypoallergenic protein fragments lose part of their native structure, in most studies, they are shown to induce an allergenspecific $\lg G$ response upon immunization, which is capable of binding natural allergens [11-13, 21, 22]. The negative effect of vaccination is the possibility of induction of Type IV allergic reaction [11-13].

The genetic transformation of allergens into a hypoallergenic immunogen can be traced using the case-study of an artificial fusion protein derived from the allergens of European house dust mite, Dermatophagoides pteronyssinus (Der p). This therapeutic allergen contains the antigenic regions of the allergens Der $p 1$, Der $p 2$, Der $p 7$ and Der $p 10$ in one molecule. Although specific Bcell epitopes are present in the fusion protein, the position and orientation of the epitopes in the new structure has changed, which affects the reactivity strength with IgE. Reducing allergenicity and increasing immunogenicity is also promoted by the increased aggregation ability of the obtained protein. In published studies, the experimental allergen binds IgE weaker compared with the recombinant unmodified allergens Der $p$ 1, Der p 2 and the whole extract of Der p. An increase in the level of blocking IgGs was noted in mice upon their immunization [22].

\section{Trend 2: T cell epitope-based allergy vaccines}

So far, two main problems have hindered the use of these vaccines:

1) The need in a large selection of various epitopes to ensure their binding to major histocompatibility complex (MHC) class II proteins. MHCII molecules have a high degree of polymorphism; in humans, they are recognized as human leukocyte antigens (HLA), and hundreds of allelic variants of both $\mathrm{MHCl}$ and $\mathrm{MHCll}$ molecules are known. In general, most people have T cells that respond in vitro to multiple epitopes within the same allergen. However, T-cell crossreactivity profile of each person is unique, and usually 1 or 2 epitopes dominate $[18,20]$;

2) The need to identify immunodominant epitopes capable of causing the secretion of cytokines in immunocompetent cells [18].

Both of these problems are currently resolvable. It has been determined that different HLA molecules can bind similar amino acid sequences of peptides. Researchers have combined HLA molecules with comparable peptide-binding features into groups known as HLA supertypes. There are HLA supertypes covering the majority of the people in the population. On the other hand, there are universal peptides able to bind to several HLA supertypes, which are preferred when creating vaccines $[18,23,24]$. These strategies enable the development of T-cell epitope vaccines with high population coverage using a limited number of peptides. Functionally, immunodominant peptides are identified in vitro by their ability to cause proliferation and secretion of cytokines (interferon- $\gamma$, IL-10 and IL-13), and by their reduced or absent IgE binding ability. Many studies searched for detecting the binding of a particular T-cell epitope of an allergen to the production of a certain interleukin. This approach can lead to a targeted distortion 
of the immune response $[18,20,24]$. At the same time, there is evidence that some CD4 T-cell epitopes stimulate specific subsets of Th [18]. T-cell epitopes that have passed this double selection constitute the basis for vaccine peptides. It is also possible to use simplified methods, such as involving the vaccines based on all available T-cell epitopes of a given allergen, but, in this particular case, the likelihood of IgE-mediated reactions increases.

The immunological aspects of such vaccination are not fully understood. It is acknowledged that immunization with T-cell epitopes of allergens leads to the induction of T-cell tolerance. Studies have noted an increase in the effects of Treg, augmented IL10 production, an increase in the Th1/Th2 ratio, and a subsequent long-term effect, associated with a change in the phenotype of allergen-specific memory $T$ cells. Although B cells remain intact during immunotherapy, some studies admitted the induction of allergen-specific IgG to T-epitope vaccines. This fact indicates the presence of preserved antibody epitopes in these vaccines $[11,14,20,24-26]$. Compared with ASIT and sublingual immunotherapy (SLIT), immunization with immunodominant T-cell epitope peptides promotes the absence of immediate IgEdependent reactions $[11,27,28]$ and provides sustained clinical benefits after a relatively short treatment course [26, 29]. In connection with preserving T-cell epitopes of the allergen, there remains the possibility of inducing Type IV allergic reaction. Worldwide development of vaccines based on T-cell epitopes is hampered by HLA polymorphism, since its variants differ significantly in various ethnic groups $[11,18]$. The case-study of a recombinant T-cell epitope vaccine, evaluating a possibility of purposefully distorting the immune response, involved five short peptides derived from the main allergen of olive pollen. These peptides have been identified as immunodominant T-cell epitopes. Blood sample investigation in the patients suffering from IgEmediated reactions to olive pollen revealed the lack of ability of all five peptides in various combinations to induce the activation of basophils in vitro. Two of the studied peptides exhibited an ability to inhibit the proliferative response to the allergen extract by $30 \%$. For the same peptides, in contrast to the allergen extract, statistically significant induction of IL-10 and IL-35 secretion in the supernatants of cultures of peripheral blood mononuclear cells was found in allergic patients. The result proved the immunoregulatory capability of these two peptides and indicated that they were top preferred candidates for vaccine with an objective of forming tolerance to allergens in patients [30].

Trend 3: B cell epitope-based and highly immunogenic carrier-protein-based allergy vaccines

To develop such vaccines, the genetic material of hypoallergenic peptides of B-cell epitopes is stitched with the genetic material of non-allergenic and highly immunogenic carrier proteins. The latter contribute to the induction of a controlled protective humoral IgG-mediated immune response against both the allergen and the carrier protein. As a result, a given fusion (chimeric) protein is obtained using recombinant technologies [1113, 31-35]. The mechanism of the immune response in the course of vaccination with these proteins is based on the hapten-carrier principle. $B C R s$ of $B$ cells specifically recognize and bind the remaining portion of the B-cell epitope of the allergen. The B-cell processes and presents a fusion protein molecule to the antigenspecific type $2 \mathrm{~T}$ helper cell in the form of a carrier protein peptide in conjunction with $\mathrm{MHClI}$. The latter helps the production of IgG antibodies. With such approach towards ASIT, systemic anaphylactic reactions and late phase of T-cell reactivity do not occur [4, 34, 36]. Experimental studies have shown that immunization of mice and rabbits with such vaccines promoted the development of specific IgG-blocking antibodies in them. In in vitro reactions, blocking IgG antibodies are able to competitively block the binding of the native allergen to the IgE of patients sensitized to the allergen. Such approach in immunotherapy enables development of combination vaccines for treatment and prevention of both allergies and infectious diseases [32, 34, 36, 37].

The recombinant vaccine, based on B-cell epitopes (BM32), for allergen-specific immunotherapy of allergy to Timothy grass pollen is interesting in its structure. This vaccine is adsorbed onto aluminum hydroxide. It contains recombinant, cross-linked, allergen-derived proteins, composed of non-allergenic peptides of B-cell epitopes (of four major Timothy-grass pollen allergens) and the surface protein of human hepatitis B virus preS domain. A randomized, double-blind, placebo-controlled ASIT study was conducted. The two-year ASIT consisted of two courses of injections: three pre-season injections of $\mathrm{BM} 32$ and a single injection in the first year of therapy after the pollen season of Timothy grass. Two-year ASIT induced an increase in the allergenspecific IgG4 response; moreover, after the second year of therapy, it was more pronounced than after the first year. No increase in proinflammatory cytokine reactions was observed. The patients, treated with BM32, exhibited reduction in allergenspecific IgE increase, induced by seasonal exposure to grass pollen, while overall improvement of their clinical condition was noted. Besides, BM32 vaccination did not cause a corresponding surge in T-cell responses to native grass pollen allergens [37, 38].

\section{Encapsulated allergens}

Some vaccines, created by scientists, can hardly be attributed to the trends described in this review. At the same time, they may be very promising at ASIT prevention. It is possible that the mechanism of action of these therapeutic allergens is different from the mechanism of action of the above vaccines, although more research is needed to understand how desensitization occurs in each case. In these therapeutic allergens, the surface structures of the protein are screened, which prevents the binding of the allergen both with immunoglobulins and B cells. Such preparation does not cause IgE-mediated allergic reactions but, rather, is successfully captured by antigen-presenting cells (APCs) and, therefore, is capable of causing a change in the nature of the immune response to an allergen during immunotherapy $[39,40]$.

Here is just an example of a vaccine created at the M.M. Shemyakin - Yu.A. Ovchinnikov Institute of Bioorganic Chemistry, Russian Academy of Sciences. Recombinant proteins of American house dust mite Dermatophagoides farinae (Der $f 1$ and Der f 2) were immersed in a chitosan matrix and then formed a polymer shell. The resulting nanoparticles of the core-shell type made it possible to shield the recombinant proteins contained in them from recognition by specific antibodies. Using sera from patients with IgE-mediated allergic reactions to house dust mites, it was proved that the resulting vaccine did not bind allergen-specific IgE antibodies [40]. It is worth noting that convenient size of the nanoparticles (90-140 nm) ensures their rapid and complete phagocytosis, which is a necessary condition for both immunogenicity and safety of the allergen. The cellular response 
to this encapsulated allergen, as measured by the proliferation of splenocytes from immunized mice, and the change in IgG levels are comparable with responses to the recombinant protein. Upon completion of the experimental and clinical study, the developed medication may be used for ASIT in higher concentrations than native allergens, which could shorten the treatment period [39, 40].

\section{DNA vaccines}

DNA vaccination has great potential as an effective preventive and therapeutic solution for ASIT. Compared to conventional protein-based vaccines, DNA vaccines are more stable, economical, easier to manufacture, and safer to handle. The classical methods of vaccine administration are intramuscular, intradermal, and subcutaneous injections, which are primarily targeted to myocytes and keratinocytes, as well as APC [41, 42]. It is believed that antigen presentation during DNA vaccination may occur via three possible mechanisms:

1) Plasmid DNA is expressed in somatic cells (e.g., myocytes), which, in turn, can generate and release antigen. This exogenous antigen can be absorbed by APC and then presented in conjunction with $\mathrm{MHCll}$ to $\mathrm{CD} 4+\mathrm{T}$ cells;

2) APCs (e.g., dendritic cells), attracted to the injection site, can also be transfected with plasmid DNA. The synthesized endogenous antigens are processed in proteasomes and presented by $\mathrm{MHCl}$ to cytotoxic T lymphocytes (CD8+ T cells);

3) The transfected somatic cells may undergo apoptosis. The apoptotic bodies are captured up by APCs, and the exogenous antigen, in conjunction with $\mathrm{MHCll}$, causes a CD4+ T cell response.

DNA vaccination studies have shown that the activation of CD8+ cytotoxic $T$ lymphocytes is largely dependent on dendritic cells, rather than being induced by somatic cells [41, 42, 43]. This presentation shapes the appropriate pattern of cytokine regulation of the immune response. In addition to the Th2 response, due to the presentation of the $\mathrm{MHCl}$ antigen, clonal expansion of cytotoxic $T$ lymphocytes also occurs. Th0 cells are transformed into Th1, producing interferon gamma and IL2. The above immunological mechanisms with existing pronounced skew of the Th1/Th2 ratio towards Th2 in patients with IgE-mediated allergic reactions should contribute to the formation of a change in the immunological response to the allergen and the synthesis of specific IgG [4]. However, after DNA vaccination, low antibody production is often found in humans and large animals [42,44].

Various methods are used to enhance the immunogenicity of DNA vaccines [42, 44-46]. One of the methods was demonstrated using the example of preparing a vaccine from the DNA of immunodominant epitopes CryJ1 or CryJ2 of Japanese cedar pollen allergens with lysosomal-associated membrane protein 1 (LAMP1), a resident protein of lysosomes. Incorporation of LAMP-1 lysosomal vector sequences into DNA plasmids directs the immunogen from the proteasome pathway to the lysosomal pathway. As a result, the allergen appears on the APC membrane in a complex with $\mathrm{MHCll} \mathrm{CD} 4+$ lymphocytes, which significantly enhances the immunogenicity of these antigens. Targeting proteins into lysosomes for degradation reduces the secretion of the allergen into the extracellular space and thereby reduces the likelihood of allergic reactions.

Immunization of mice with the CryJ-lysosomal-associated membrane protein (CryJ-LAMP) DNA vaccine resulted in development of high levels of specific IgG2a antibodies (anti-CryJ1 or anti-CryJ2), moderate levels of IgG1 antibodies and low levels of IgE antibodies. Experimental results have also proven that vaccination with CryJ2-LAMP can suppress basophil activation and/or function, and induce CD4+ T cell memory. At the same time, the DNA vaccine remains effective in stimulating a strong response of $\mathrm{CD} 8+\mathrm{T}$ cells, which is apparently associated with the possibility of a partial allergen degradation in proteasomes and its subsequent presentation by $\mathrm{MHCl}$ into APC. The latter contributes to the production of high levels of interferon gamma and, accordingly, distortion of the immune response towards Th1. Experimental models have shown that the immunological effects of such DNA vaccines were not associated with the classical mechanism of ASIT action, specifically with regulatory $T$ cells (Tregs) and IL-10 [47]. However, some publications present the data implying that DNA vaccine with a lysosomal-associated membrane protein is able to induce Tregs [48].

Understanding the action mechanism of DNA vaccines in atopic conditions would enable their wider use in the future for the prevention of sensitization in patients with atopy to new allergens, rather than solely for the treatment of patients with allergic pathology $[48,49]$.

\section{Conclusion}

To date, the structures of most allergens causing allergic reactions have already been discovered. This fact has allowed the development of several novel approaches towards creation of recombinant allergy vaccines. In experimental animal models and in clinical trials on a limited contingent of patients, it has been shown that the new generation of allergy vaccines, in most cases, dramatically reduces systemic side effects, is highly effective and safe for specific immunotherapy, and allows achieving positive therapeutic results in a short time [37, 38, 50]. In addition, contemporary allergy vaccines can be promising therapeutic agents for prophylaxis in individuals predisposed to IgE-mediated allergic diseases [11].

\section{Conflict of interest}

None declared.

\section{References}

1. Akdis CA, Akdis M. Mechanisms of allergen-specific immunotherapy and immune tolerance to allergens. World Allergy Organ J 2015; 8(1): 17. https://doi.org/10.1186/s40413-015-0063-2.

2. Petrova SYu, Khlgatian SV, Berzhets VM, Radikova OV. Modern concept of pathogenesis of atopic diseases. Immunopathology, Allergology, Infectology 2019; (1): 72-79. Russian. https://doi.org/10.14427/jipai.2019.1.72

3. Fujita $H$, Soyka MB, Akdis M, Akdis CA. Mechanisms of allergen-specific immunotherapy. Clin Transl Allergy 2012; 2(1): 2 https://doi.org/10.1186/2045-7022-2-2.

4. Petrova SYu, Berzhets VM, Petrova NS, Hrulyova VA, Emelyanova OYu, Hlgatyan SV, et al. Future prospect of allergens' medical forms. From abstract problems to concrete solutions. Immunopathology, Allergology, Infectology 2018; (1): 40-47. Russian. https://doi.org/10.14427/jipai.2018.1.40.

5. Jutel $M$, Akdis CA. Immunological mechanisms of allergen-specific immunotherapy. Allergy 2011; 66(6): 725-732. https://doi.org/10.1111/j.1398-9995.2011.02589.x. 
6. Soliman M, Ellis AK. The role of synthetic peptide immuno-regulatory epitope (SPIRE) in the treatment of allergic disease. Curr Treat Options Allergy 2017; 4(1): 22-29. https://doi.org/10.1007/s40521-017-0115-7.

7. Grönlund $H$, Gafvelin $G$. Recombinant Bet $\vee 1$ vaccine for treatment of allergy to birch pollen. Hum Vaccin 2010; 6(12): 970-977. https://doi.org/10.4161/hv.6.12.13348.

8. Chen KW, Blatt K, Thomas WR, Swoboda I, Valent P, Valenta R, et al. Hypoallergenic Der $p$ 1/Der $p 2$ combination vaccines for immunotherapy of house dust mite allergy. J Allergy Clin Immunol 2012; 130(2): 435-443.e4. https://doi.org/10.1016/j.jaci.2012.05.035.

9. Valenta R, Ferreira F, Focke-Tejkl M, Linhart B, Niederberger V, Swoboda I, et al. From allergen genes to allergy vaccines. Annu Rev Immunol 2010; 28: 211-241. https://doi.org/10.1146/annurevimmunol-030409-101218.

10. Valenta R. The future of antigenspecific immunotherapy of allergy. Nat Rev Immunol 2002; 2(6): 446-453. https://doi.org/10.1038/nri824

11. Marth K, Focke-Tejkl M, Lupinek C, Valenta R, Niederberger V. Allergen Peptides, Recombinant Allergens and Hypoallergens for AllergenSpecific Immunotherapy. Curr Treat Options Allergy 2014; 1(1): 91-106. https://doi.org/10.1007/s40521-013-0006-5.

12. Valenta R, Niespodziana K, Focke-Tejkl M, Marth $\mathrm{K}$, Huber $\mathrm{H}$, Neubauer A. Recombinant allergens: What does the future hold? J Allergy Clin $\begin{array}{lll}\text { Immunol 2011; } & \text { 860-864. }\end{array}$ https://doi.org/10.1016/i.jaci.2011.02.016.

13. Valenta R, Linhart B, Swoboda I, Niederberger V. Recombinant allergens for allergen-specific immunotherapy: 10 years anniversary of immunotherapy with recombinant allergens. Allergy 2011; 66(6): 775783. https://doi.org/10.1111/j.1398-9995.2011.02565.x.

14. Akdis M. New treatments for allergen immunotherapy. World Allergy Organ J 2014; 7(1): 23. https://doi.org/10.1186/1939-4551-7-23.

15. Svirshchevskaya EV, Alekseeva LG, inventors; Svirshchevskaya EV, Alekseeva LG, assignee. Heterologous peptide mini-antigene in polymer particle for making anti-allergy vaccine. Russian patent RU $2480 \quad 479 . \quad 2011 \quad$ Nov 1. http://www.freepatent.ru/images/patents/471/2480479/patent2480479.pdf

16. Kringelum JV, Nielsen M, Padkjær SB, Lund O. Structural analysis of Bcell epitopes in antibody: protein complexes. Mol Immunol 2013; 53(12): 24-34. https://doi.org/10.1016/j.molimm.2012.06.001.

17. Jespersen MC, Peters B, Nielsen M, Marcatili P. BepiPred-2.0: improving sequence-based B-cell epitope prediction using conformational epitopes. Nucleic Acids Res 2017; 45(W1): W24-W29. https://doi.org/10.1093/nar/gkx346.

18. Sanchez-Trincado JL, Gomez-Perosanz M, Reche PA. Fundamentals and Methods for T- and B-Cell Epitope Prediction. J Immunol Res 2017; 2017: 2680160. https://doi.org/10.1155/2017/2680160.

19. Tolar P, Spillane KM. Force generation in B-cell synapses: mechanisms coupling B-cell receptor binding to antigen internalization and affinity discrimination. Adv Immunol 2014; 123: 69-100. https://doi.org/10.1016/b978-0-12-800266-7.00002-9.

20. Woodfolk JA. T-cell responses to allergens. J Allergy Clin Immunol 2007; 119(2): 280-294. https://doi.org/10.1016/j.jaci.2006.11.008

21. Sircar G, Jana K, Dasgupta A., Saha S, Bhattacharya S. Epitope mapping of Rhi $\circ 1$ and generation of a hypoallergenic variant: a candidate molecule for fungal allergy vaccines. J Biol Chem 2016; 291(34): 1801618029. https://doi.org/10.1074/jbc.m116.732032.

22. Martínez D, Munera M, Cantillo JF, Wortmann J, Zakzuk J, Keller W, et al. An Engineered Hybrid Protein from Dermatophagoides pteronyssinus Allergens Shows Hypoallergenicity. Int J Mol Sci 2019; 20(12): 3025. https://doi.org/10.3390/ijms20123025.

23. Greenbaum J, Sidney J, Chung J, Brander C, Peters B, Sette A. Functional classification of class II human leukocyte antigen (HLA) molecules reveals seven different supertypes and a surprising degree of repertoire sharing across supertypes. Immunogenetics 2011; 63(6): 325-335. https://doi.org/10.1007/s00251-011-0513-0.
24. Worm M, Lee HH, Kleine-Tebbe J, Hafner RP, Laidler P, Healey D, et al. Development and preliminary clinical evaluation of a peptide immunotherapy vaccine for cat allergy. J Allergy Clin Immunol 2011; 127: 89-97. https://doi.org/10.1016/j.jaci.2010.11.029.

25. Sancho Al, Wallner M, Hauser M, Nagl B, Himly M, Asam C, et al. T cell epitope-containing domains of ragweed $A m b$ a 1 and mugwort Art $v 6$ modulate immunologic responses in humans and mice. PLoS One 2017 12(1): e0169784. https://doi.org/10.1371/journal.pone.0169784

26. Patel $D$, Couroux $P$, Hickey $P$, Salapatek $A M$, Laidler $P$, Larché $M$, et al. Fel $d$ 1-derived peptide antigen desensitization shows a persistent treatment effect 1 year after the start of dosing: a randomized, placebo-controlled study. J Allergy Clin Immunol 2013; 131(1): 103109.e1-7. https://doi.org/10.1016/j.jaci.2012.07.028.

27. Pellaton C, Perrin Y, Boudousquie C, Barbier N, Wassenberg J, Corradin $\mathrm{G}$, et al. Novel birch pollen specific immunotherapy formulation based on contiguous overlapping peptides. Clin Transl Allergy 2013; 3(1): 17. https://doi.org/10.1186/2045-7022-3-17.

28. Tonti $E$, Larché $M$. Concepts and perspectives on peptide-based immunotherapy in allergy. Allergo Journal International 2016; 25(6): 144-153. http://doi.org/10.1007\%2Fs40629-016-0126-0.

29. Hafner RP, Couroux $P$, Salapatek $A$, Hickey $P$, Laidler $P$, Larché $M$, et al. Immunotherapy - 2080. Fel d 1 derived peptide antigen desensitization results in a persistent treatment effect on symptoms of cat allergy 1 year after 4 doses. World Allergy Organization Journal 2013; 6(Suppl 1): P162. https://doi.org/10.1186/1939-4551-6-S1-P162.

30. Calzada D, Cremades-Jimeno L, Pedro MÁ, Baos S, Rial M, Sastre J, et al. Therapeutic potential of peptides from Ole e 1 in olive-pollen allergy. Sci Rep 2019; 9(1): 15942. https://doi.org/10.1038/s41598019-52286-3.

31. Focke-Tejkl M, Weber M, Niespodziana K, Neubauer A, Huber $H$, Henning $R$, et al. Development and characterization of a recombinant, hypoallergenic, peptide-based vaccine for grass pollen allergy. J Allergy Clin Immunol 2015; 135(5): 1207-1217.e11. https://doi.org/10.1016/j.jaci.2014.09.012.

32. Twaroch TE, Focke M, Fleischmann K, Balic N, Lupinek C, Blatt K, et al. Carrier-bound Alt a 1 peptides without allergenic activity fo vaccination against Alternaria alternata allergy. Clin Exp Allergy 2012; 42(6): 966-975. https://doi.org/10.1111/j.1365-2222.2012.03996.x.

33. Edlmayr J, Niespodziana K, Focke-Tejkl M, Linhart B, Valenta R. Allergen-specific immunotherapy: towards combination vaccines for allergic and infectious diseases. Curr Top Microbiol Immunol 2011; 352: 121-140. https://doi.org/10.1007/82_2011_130.

34. Marth K, Breyer I, Focke-TejkI M, Blatt K, Shamji MH, Layhadi J, et al. A nonallergenic birch pollen allergy vaccine consisting of hepatitis PreSfused Bet $v 1$ peptides focuses blocking IgG toward IgE epitopes and shifts immune responses to a tolerogenic and Th1 phenotype. J Immunol 2013; 190(7): 3068-3078. https://doi.org/10.4049/jimmunol.1202441.

35. Niespodziana K, Focke-TejkI M, Linhart B, Civaj V, Blatt K, Valent P, et al. A hypoallergenic cat vaccine based on Fel $d$ 1-derived peptides fused to hepatitis B PreS. J Allergy Clin Immunol 2011; 127(6): 15621570.e6. https://doi.org/10.1016/j.jaci.2011.02.004.

36. Twaroch TE, Focke M, Civaj V, Weber M, Balic N, Mari A, et al. Carrierbound, nonallergenic Ole e 1 peptides for vaccination against olive pollen allergy. J Allergy Clin Immunol 2011; 128(1): 178-184.e7. https://doi.org/10.1016/j.jaci.2011.03.011.

37. Zieglmayer $P$, Focke-Tejkl $M$, Schmutz $R$, Lemell $P$, Zieglmayer $R$, Weber M, et al. Mechanisms, safety and efficacy of a B cell epitopebased vaccine for immunotherapy of grass pollen allergy. EBioMedicine 2016; 11: 43-57. https://doi.org/10.1016/j.ebiom.2016.08.022.

38. Eckl-Dorna J, Weber M, Stanek V, Linhart B, Ristl R, Waltl EE, et al. Two years of treatment with the recombinant grass pollen allergy vaccine BM32 induces a continuously increasing allergen-specific IgG response. 
EBioMedicine 2019; 50: 421-432. https://doi.org/10.1016/j.ebiom.2019.11.006.

39. Kashirina E, Reshetov P, Alekseeva L, Berzhets V, Ryazantsev D, Zubov $V$, et al. Encapsulation of Allergens into Chitosan-Alginate Nanoparticles Prevents IgE Binding. J J Vaccine Vaccination 2015; 1(3): 012.

https://www.academia.edu/37888094/Encapsulation_of_Allergens in to Chitosan Alginate Nanoparticles Prevents IgE Binding.

40. Kashirina El, Reshetov PD, Alekseeva LG, Ryazantsev DY, Zubov VP, Guryanova SV, et al. Capsulation of House-Dust-Mite Allergens into Nanoparticles Developed from Chitosan and Alginate. Nanotechnologies in Russia 2015; 10(7-8): 627-635 https://doi.org/10.1134/S1995078015040084.

41. Hobernik D, Bros M. DNA vaccines - How far from clinical use? Int $J$ Mol Sci 2018; 19(11): 3605. https://doi.org/10.3390/ijms19113605.

42. Goryaev AA, Savkina MV, Obukhov Yu I, Merkulov VA, Olefir YuV. DNA and RNA vaccines: current status, quality requirements and specific aspects of preclinical studies. BIOpreparations. Prevention, Diagnosis, Treatment 2019; 19(2): 72-80. Russian. https://doi.org/10.30895/2221-996X-2019-19-2-72-80.

43. Burmester GR, Pezzutto A. Naglyadnaya immunologiya. 4rd ed. Moscow, Russia: Laboratoriya znaniy, 2018: 64-67.

44. Lu S. Immunogenicity of DNA vaccines in humans: It takes two to tango. Hum Vaccin 2008; 4(6): 449-452. https://doi.org/10.4161/hv.4.6.6179.

45. Marques ET Jr, Chikhlikar P, de Arruda LB, Leao IC, Lu Y, Wong J, et al. HIV-1 p55Gag encoded in the lysosome-associated membrane protein1 as a DNA plasmid vaccine chimera is highly expressed, traffics to the major histocompatibility class II compartment, and elicits enhanced immune responses. J Biol Chem 2003; 278(39): 37926-3736. https://doi.org/10.1074/jbc.m303336200.

46. Graham BS, Enama ME, Nason MC, Gordon IJ, Peel SA, Ledgerwood JE, et al. DNA vaccine delivered by a needle-free injection device improves potency of priming for antibody and CD8+ T-cell responses after rAd5 boost in a randomized clinical trial. PLoS One 2013; 8(4): e59340. https://doi.org/10.1371/journal.pone.0059340.

47. Su Y, Connolly M, Marketon A, Heiland T. CryJ-LAMP DNA vaccines for Japanese Red Cedar allergy induce robust Th1type immune responses in murine model. J Immunol Res 2016 ;2016: 4857869. https://doi.org/10.1155/2016/4857869.

48. Weinberger EE, Isakovic A, Scheiblhofer S, Ramsauer C, Reiter K, Hauser-Kronberger $C$, et al. The influence of antigen targeting to subcellular compartments on the anti-allergic potential of a DNA vaccine.
Vaccine
2013;
31(51):
6113-6121. https://doi.org/10.1016/j.vaccine.2013.08.005.

49. Zhu Z, Yu J, Niu Y, Sun S, Liu Y, Saxon A, et al. Prophylactic and therapeutic effects of polylysine-modified Ara $\mathrm{h} 2$ DNA vaccine in a mouse model of peanut allergy. Int Arch Allergy Immunol 2017; 171(34): 241-250. https://doi.org/10.1159/000453264.

50. Soliman M, Ellis AK. The role of synthetic peptide immuno-regulatory epitope (SPIRE) in the treatment of allergic disease. Curr Treat Options Allergy 2017; 4(1): 22-29. https://doi.org/10.1007/s40521-017-0115-7.

\section{Authors:}

Stanislava Yu. Petrova - MD, PhD, Senior Researcher, Laboratory of Allergens, I.I. Mechnikov Research Institute for Vaccines and Sera, Moscow, Russia. https://orcid.org/0000-0003-3034-0148.

Svetlana V. Khlgatian - PhD, Principal Researcher, Laboratory of Allergens, I.I. Mechnikov Research Institute for Vaccines and Sera, Moscow, Russia; Physician in Clinical Laboratory Diagnostics, Research Center of Neurology, Moscow, Russia. https://orcid.org/0000-0001-8354-7682.

Elena V. Svirshchevskaya - PhD, Senior Researcher, Laboratory of Cell Interactions, M.M. Shemyakin - Yu.A. Ovchinnikov Institute of Bioorganic Chemistry, Russian Academy of Sciences, Moscow, Russia. https://orcid.org/0000-0002-5647-9298.
Anna V. Vasilyeva - Research Fellow, Laboratory of Allergens, I.I. Mechnikov Research Institute for Vaccines and Sera, Moscow, Russia. https://orcid.org/0000-0002-7703-2698.

Valentina M. Berzhets - DsC, Professor, Head of the Laboratory of Allergens, I.I. Mechnikov Research Institute for Vaccines and Sera, Moscow, Russia. https://orcid.org/0000-0001-5055-7593. 